\title{
SOLVABILITY OF ORDINARY DIFFERENTIAL EQUATIONS NEAR SINGULAR POINTS
}

\author{
BY \\ HOMER G. ELLIS
}

1. Introduction. If the point $(0, b)$ is critical for the differential equation $f^{\prime}(x)=G(x, f(x))$ by virtue of the fact that $G$ is sufficiently singular near $(0, b)$ that $G(0, b)$ may not be defined in such a way as to make $G$ continuous there, then there arises the problem of finding a condition on $G$ which will ensure that some solution $f$ will satisfy $f(0+)=b$, so that the corresponding integral curve will have a terminus at $(0, b)$. Such a condition is presented here. In the special case where $G(x, w)=Q(x, w) / P(x, w)$, with $P$ and $Q$ analytic at $(0, b)$, it is approximately equivalent to existence of a critical direction at $(0, b)$.

The problem can be formally reduced to the case in which $b=0$, and is then tantamount to investigating the improper integral equation

$$
f=\int_{0+} G(x, f(x)) d x .
$$

By way of illustrating the ideas which are to follow, suppose that there exist a function $u$ on a half-open interval $(0, a]$ and a function $K$ such that, whenever $t \in \operatorname{dom} K, \lim _{x \rightarrow 0} \int_{0+}^{x} G(y, t u(y)) d y / u(x)=K(t)$-as a case in point, let $G(x, w)$ $=1+c(w / x)^{2}, c$ being a constant, let $u(x)=x$, and let $K(t)=1+c t^{2}$. Consider now any function $f$ for which there is a number $t$ in the domain of $K$ such that $\lim _{x \rightarrow 0} f(x) / u(x)=t$. If this convergence is rapid enough, then

$$
\lim _{x \rightarrow 0} \int_{0+}^{x} G(y, f(y)) d y / u(x)=K(t),
$$

for when $y$ is small, $t u(y)$ is a good approximation to $f(y)$. But if $f$ is a solution of (1), then $\lim _{x \rightarrow 0} \int_{0+}^{x} G(y, f(y)) d y / u(x)=\lim _{x \rightarrow 0} f(x) / u(x)=t$, and therefore $t=K(t)$.

The promised condition upon $G$ sufficient to ensure solvability of (1) will be stated in terms of existence of functions similar to $u$ and $K$, and existence of a solution of an inequality which takes the place of the equation $t=K(t)$. This condition will be shown to be necessary for the solvability of (1) in many cases of interest, as well as sufficient. Also, a certain condition counter to the one mentioned, together with unsolvability of the corresponding inequality, will be shown to imply in many instances that (1) is unsolvable. By use of the latter result it can be

Presented to the Society, January 24, 1962 under the title Solutions of first order differential equations, including singular cases; received by the editors February 21, 1964. 
seen for the example mentioned above that (1) has no real-valued solution if $c>1 / 4$, since $1+c t^{2} \leqq t$ then has no real solution.

The problem can be thought of as an initial-value problem in which a person, standing at the point $(0, b)$ and trying to find an integral curve to stroll along, narrows the search to those paths which are asymptotic to multiples of $u$, and then checks the multipliers for suitability.

When the problem is looked at as an initial-value problem, the considerations to be set forth here are seen to fall into a line of inquiry which over the years has arrested the attention of many mathematicians, including notably Cauchy [4], Lipschitz [5], Peano [7], de La Vallée-Poussin [6, p. 43] and Carathéodory [3].

2. A hypothesis about $G$. Suppose that $G$ is a function whose values are in $P_{\infty}$, the compact complex plane, and whose domain is $(0, \infty) \times D$, where $D=P$ or $D$ is a circular disc in $P$, either open or closed, and centered at $0, P$ denoting the noncompact complex plane. Let $I$ denote the interval common to $D$ and the interval $[0, \infty)$.

If $a>0$ and it is stated that a function, $h$ say, whose domain is the interval $(0, a]$ is integrable from $0+$ to $x$, where $x \in(0, a]$, this will mean that if $0<\delta \leqq x$, then $h$ is Lebesgue-integrable from $\delta$ to $x$ and $\lim _{\delta \rightarrow 0} \int_{\delta}^{x} h$ exists and is finite; this limit will be denoted by $\int_{0+}^{x} h$.

Consider now the following hypothesis about $G$, in which, as in all that follows, both $\infty-\infty$ and $\infty \cdot 0$ are taken to be 0 .

HYPOTHESIS A. There exist a finite positive number $a$, a function $G_{0}:(0, a] \rightarrow P_{\infty}$, a finite-valued nonnegative function $u$ on $(0, a]$, a finite-valued nonnegative function $K_{0}$ on $(0, a]$ and a nonnegative function $H$ on $(0, a] \times I$, such that

(i) $G_{0}$ is integrable from $0+$ to $a$,

(ii) if $x \in(0, a]$, then

$$
\left|\int_{0+}^{x} G_{0}\right| \leqq K_{0}(x) u(x),
$$

(iii) (a) for each number $x$ in $(0, a], H(x, r)$ is nondecreasing in $r$, (b) if $x \in(0, a]$ and $w \in D$, then

$$
\left|G(x, w)-G_{0}(x)\right| \leqq H(x,|w|),
$$

and

(c) for at least one nonnegative number $t$ such that $t u(x) \in I$ when $x \in(0, a], H(x, t u(x))$ as a function of $x$ is integrable from $0+$ to $a$, and

(iv) with $J$ signifying the set of which $t$ is an element only if $t \geqq 0$ and $H(x, t u(x))$ as a function of $x$ is integrable from $0+$ to $a$, there exists a finitevalued nonnegative function $K$ on $(0, a] \times J$ such that if $x \in(0, a]$ and $t \in J$, then

$$
\int_{0+}^{x} H(y, t u(y)) d y \leqq K(x, t) u(x) .
$$


A few examples will perhaps help with the assimilation of this hypothesis. They are the following.

EXAmple 1. This is the example of the introduction. Let $D=P$, let $c$ be a complex number, and as before let $G(x, w)=1+c(w / x)^{2}$. Then $I=[0, \infty)$ and the truth of Hypothesis $A$ is confirmed by taking $a$ to be any finite positive number and defining functions $G_{0}, u, K_{0}, H$ and $K$ so that $G_{0}(x)=G(x, 0)=1$, $u(x)=\int_{0+}^{x} G_{0}=x, K_{0}(x)=1, H(x, r)=|c|(r / x)^{2}$, and $K(x, t)=|c| t^{2} . J$ then turns out to be the interval $[0, \infty)$.

EXAmple 2. Let $D=P$, and let $G(x, w)=x+w / x$. Again $I=[0, \infty)$, and if $m$ is any finite nonnegative number, then the conditions of Hypothesis $\mathrm{A}$ are satisfied if $0<a<\infty, G_{0}(x)=G(x, 0)=x, u(x)=2 \int_{0+}^{x} G_{0}+m x=x^{2}+m x$, $K_{0}(x)=\left(\frac{1}{2} x^{2}\right) /\left(x^{2}+m x\right), H(x, r)=r / x$, and $K(x, t)=\left[\left(\frac{1}{2} x^{2}+m x\right) /\left(x^{2}+m x\right)\right] t$. Here also $J=[0, \infty)$.

ExAmple 3. Let $D=P$, and let $G(x, w)=x+(|w| / x)^{1 / 2}$. Then $I=[0, \infty)$ and Hypothesis A is confirmed if $0<a<\infty, G_{0}(x)=G(x, 0)=x, u(x)=2 \int_{0+}^{x} G_{0}$ $=x^{2}, K_{0}(x)=\frac{1}{2}, H(x, r)=(r / x)^{1 / 2}$, and $K(x, t)=\left(2 / 3 x^{1 / 2}\right) t^{1 / 2}, J$ being the interval $[0, \infty)$.

EXAMPLE 4. Let $D$ and $G$, and therefore $I$, be the same as in Example 3. Let $a$ be any finite positive number, $G_{0}(x)=G(x, 0)=x, u(x)=x, K_{0}(x)=\frac{1}{2} x, H(x, r)$ $=(r / x)^{1 / 2}$, and $K(x, t)=t^{1 / 2}$. Then $J=[0, \infty)$ and the conditions of Hypothesis $A$ are met by these functions.

Notice that in each of these example all three possible equalities in the statement of Hypothesis A are satisfied. For (3) this results in part from the happenstance that in each example $\left|G(x, w)-G_{0}(x)\right|=\left|G(x,|w|)-G_{0}(x)\right|$, but otherwise it is not merely a coincidence. In fact if Hypothesis $\mathrm{A}$ is true and $G_{0}, u, K_{0}, H$ and $K$ constitute one set of functions which meets its conditions, then with one possible barrier its conditions are also met by the set $G_{0}, u, K_{0}^{*}, H_{,}^{*} K^{*}$, for which

$$
\begin{aligned}
K_{0}^{*}(x)= \begin{cases}0 & \text { if } u(x)=0, \\
\frac{\left|\int_{0+}^{x} G_{0}\right|}{u(x)} & \text { if } u(x) \neq 0,\end{cases} \\
H^{*}(x, r)=\sup _{|w| \leqq r}\left|G(x, w)-G_{0}(x)\right|,
\end{aligned}
$$

and

$$
K^{*}(x, t)= \begin{cases}0 & \text { if } u(x)=0, \\ \frac{\int_{0+}^{x} H^{*}(y, t u(y)) d y}{u(x)} & \text { if } u(x) \neq 0 .\end{cases}
$$


The possible barrier is that there might not be a nonnegative number $t$ such that $t u(x) \in I$ when $x \in(0, a]$ and such that $H^{*}(x, t u(x))$ is integrable from $0+$ to $a$. In this event $H$ might still be replaceable by a function with smaller values, but it would not be minimal in the way that $H^{*}$ is.

For the new choice of $K_{0}, H$ and $K$ the set which corresponds to $J$ might be different from the original set $J$, so that the domain of $K^{*}$ and the domain of $K$ might not coincide. In each case, however, (2) and (4) become equalities, while $H^{*}$ is the smallest-valued function which satisfies (iii)(a) and (iii)(b) as $H$ does. For each of the examples cited, $K_{0}=K_{0}^{*}, H=H^{*}$, and $K=K^{*}$.

3. Solvability of $f=\int_{0+} G(x, f(x)) d x$ as a consequence of restrictedness in growth of $G$ somewhere near 0 . In the presence of Hypothesis $A$ the inequality which was promised as a replacement for the equation $t=K(t)$ is $K_{0}(x)+K(x, t)$ $\leqq t$. Theorem 1 will relate the question of existence of a function $f$ which satisfies the integral equation (1) to the question of existence of a number $t$ which satisfies this inequality for every number $x$ in the interval $(0, a]$. The following definition will help to focus attention on the essential ideas involved.

Definition 1. The function $G:(0, \infty) \times D \rightarrow P_{\infty}$ is said to be restricted in growth somewhere near 0 if and only if Hypothesis $A$ is true and for some set $\left\{G_{0}, u, K_{0}, H, K\right\}$ of functions which confirms it there is a number $t$ such that

$$
K_{0}(x)+K(x, t) \leqq t
$$

if $0<x \leqq a$.

The proof of Theorem 1 to be given will be an application of a fixed-point theorem due to Schauder [8], the principal ideas of which were developed by Brouwer [2] and Birkhoff and Kellogg [1]. As a guarantee of the existence and continuity of the mapping which is to have the fixed point, an additional restriction upon the function $G$ will be required. In Theorem 1 such a restriction will be imposed implicitly. In Theorems 2, 3 and 4 such restrictions will be made explicitly.

In all that follows, if $0<a<\infty, C_{0}(0, a]$ will denote the Banach space of all continuous functions $f:(0, a] \rightarrow P$ for which $f(0+)=0$, with

$$
\|f\|=\sup _{x \in(0, a]}|f(x)| \text {. }
$$

THEOREM 1. Suppose that

(i) $G$ is restricted in growth somewhere near 0 , and $G_{0}, u, K_{0}, H, K$ and $t$ constitute a set of functions and a number $t$ which together confirm this fact, and

(ii) $E$ denotes the set of all functions $f$ in $C_{0}(0, a]$ for which

$$
|f(q)-f(p)| \leqq\left|\int_{p}^{q} G_{0}\right|+\int_{p}^{q} H(x, t u(x)) d x
$$

if $0<p \leqq q \leqq a$. 
Then, for each function $f$ in $E, f(x) \in D$ when $x \in(0, a]$, and if $G(x, f(x))$ as a function of $x$ is measurable, it is also integrable from $0+$ to a; furthermore, if, for every function $f$ in $E, G(x, f(x))$ is measurable, and the mapping $U: E \rightarrow C_{0}(0, a]$ defined by $U f=\int_{0+} G(x, f(x)) d x$ is continuous, then $U$ has $a$ fixed point, which is a function $f$ with the property that $f=\int_{0+} G(x, f(x)) d x$ and, since it belongs to $E$, the additional property that $|f(x)| \leqq t u(x)$ if $0<x \leqq a$.

Proof. The fixed-point theorem to be applied states that if $T$ is a continuous mapping into itself of a compact and convex point set $M$ in a Banach space, then there is a point $z$ in $M$ such that $T z=z$. To begin the application let the space in question be $C_{0}(0, a]$ and let $M$ be the set $E$.

$M$ is clearly convex, for if each of $f$ and $g$ is in $E$ and $0 \leqq s \leqq 1$, then $(1-s) f+s g \in C_{0}(0, a]$ and if $0<p \leqq q \leqq a$, then

$$
\begin{aligned}
\mid((1-s) f+s g)(q) & -((1-s) f+s g)(p) \mid \\
& \leqq(1-s)|f(q)-f(p)|+s|g(q)-g(p)| \\
& \leqq\left|\int_{p}^{q} G_{0}\right|+\int_{p}^{q} H(x, t u(x)) d x .
\end{aligned}
$$

$M$ is closed by virtue of the fact that if $f$ is a function, continuous or not, and, for some number $p$ and number $q$ such that $0<p \leqq q \leqq a$, $|f(q)-f(p)|>\left|\int_{p}^{q} G_{0}\right|+\int_{p}^{q} H(x, t u(x)) d x$, then $f$ cannot be approached by functions in $E$ even in the pointwise sense, much less in the uniform sense.

To see that $M$ is compact consider for each function $f$ in $E$ its extension $f^{*}$ to the closed interval $[0, a]$ for which $f^{*}(0)=0$. Since the term on the right-hand side of (6) is independent of $f$ and approaches 0 with $q-p$, it follows that the functions of $E$ are equicontinuous with one another at each point of $(0, a]$, as therefore must be their extensions. From (6) it also follows, since $f(0+)=0$, that

$$
|f(q)| \leqq\left|\int_{0+}^{q} G_{0}\right|+\int_{0+}^{q} H(x, t u(x)) d x,
$$

and so by the same token as before the extended functions are equicontinuous with one another at 0 as well, inasmuch as $\left|f^{*}(q)-f^{*}(0)\right|=|f(q)|$. The fact that these extended functions are equicontinuous with one another at each point of the closed interval $[0, a]$ and have a common value at one such point implies, as a consequence of the Arzelà-Ascoli Theorem, that every infinite sequence of some of them has a uniformly convergent subsequence. This conclusion carries over to the functions of $E$, which are the points of $M$. $M$ therefore, being closed, is compact.

Suppose that $f \in E$. From (7), (2), (4) and the definition of $t$ it follows that if $0<x \leqq a$, then 


$$
|f(x)| \leqq K_{0}(x) u(x)+K(x, t) u(x)=\left[K_{0}(x)+K(x, t)\right] u(x) \leqq t u(x) .
$$

Since $t u(x) \in I$ when $x \in(0, a], f(x)$ must belong to $D$ when $x \in(0, a]$. Furthermore, in view of (3) and the nondecreasingness of $H(x, r)$ when $x$ is fixed,

$$
\left|G(x, f(x))-G_{0}(x)\right| \leqq H(x,|f(x)|) \leqq H(x, t u(x)) .
$$

Suppose now that $G(x, f(x))$ is measurable and $0<p \leqq q \leqq a$. Since by hypothesis $G_{0}$ is integrable from $0+$ to $a$, and therefore measurable, so is $G(x, f(x))$ $-G_{0}(x)$ measurable. $H(x, t u(x))$ is integrable from $0+$ to $a$, and as a result is integrable from $p$ to $q$. $G(x, f(x))-G_{0}(x)$ then, being measurable and dominated by a function which is integrable from $p$ to $q$, is itself integrable from $p$ to $q$, along with $\left|G(x, f(x))-G_{0}(x)\right|$. What is more, by virtue of (9),

$$
\begin{aligned}
\left|\int_{p}^{q}\left[G(x, f(x))-G_{0}(x)\right] d x\right| & \leqq \int_{p}^{q}\left|G(x, f(x))-G_{0}(x)\right| d x \\
& \leqq \int_{p}^{q} H(x, t u(x)) d x .
\end{aligned}
$$

Now because there is a finite number $m$ such that $\lim _{\delta \rightarrow 0} \int_{\delta}^{a} H(x, t u(x)) d x=m$, the Cauchy convergence criterion is met by this integral, or in other words if $\varepsilon>0$, there is a positive number $\delta$ such that

$$
\left|\int_{p}^{q} H(x, t u(x)) d x\right|<\varepsilon
$$

when $p<\delta$ and $q<\delta$. But this, in view of the last inequalities, implies that $\int_{\delta}^{a}\left[G(x, f(x))-G_{0}(x)\right] d x$ meets the convergence criterion, and therefore that there is a finite complex number $n$ such that $\lim _{\delta \rightarrow 0} \int_{\delta}^{a}\left[G(x, f(x))-G_{0}(x)\right] d x=n$, or, what is the same thing, $G(x, f(x))-G_{0}(x)$ is integrable from $0+$ to $a$. From this and the fact that $G_{0}$ is integrable from $0+$ to $a$ it follows that $G(x, f(x))$ is integrable from $0+$ to $a$, and therefore that the definition given for $U$ makes sense and does define a mapping, which by virtue of that definition maps $E$ into $C_{0}(0, a]$.

To complete the application of the fixed-point theorem let $T=U$. Since by hypothesis $U$ is continuous, there remains to be established only that $T(M) \subseteq M$. This, however, follows immediately from (10), for

$$
\begin{aligned}
|(U f)(q)-(U f)(p)| & =\left|\int_{p}^{q} G(x, f(x)) d x\right| \\
& \leqq\left|\int_{p}^{q} G_{0}\right|+\left|\int_{p}^{q}\left[G(x, f(x))-G_{0}(x)\right] d x\right| \\
& \leqq\left|\int_{p}^{q} G_{0}\right|+\int_{p}^{q} H(x, t u(x)) d x,
\end{aligned}
$$

so that $U f \in E$. 
Therefore $U$ leaves some function in $E$ fixed. This is a function $f$ such that $f=\int_{0+} G(x, f(x)) d x$, and, by virtue of $(8),|f(x)| \leqq t u(x)$ if $0<x \leqq a .-$ End of proof.

The next theorem is a corollary of Theorem 1 .

THEOREM 2. Suppose that

(i) $G$ is restricted in growth somewhere near 0 , and $G_{0}, u, K_{0}, H, K$ and $t$ constitute a set of functions and a number $t$ which together confirm this fact, and

(ii) $G(x, w)$ is measurable as a function of $x$ when $w \in D$, and for almost every number $x$ in $(0, a]$ is continuous as a function of $w$.

Then there is a function $f$ in $C_{0}(0, a]$ such that $f=\int_{0+} G(x, f(x)) d x$,

$$
|f(q)-f(p)| \leqq\left|\int_{p}^{q} G_{0}\right|+\int_{p}^{q} H(x, t u(x)) d x
$$

if $0<p \leqq q \leqq a$, and $|f(x)| \leqq t u(x)$ if $0<x \leqq a$.

Proof. (i) of the hypothesis of Theorem 1 is assumed here. (ii) is satisfied by appropriately defining $E$, and therefore the conclusion of Theorem 1 follows from the hypothesis of Theorem 2.

That for each function $f$ in $E, G(x, f(x))$ is measurable can be established by means of a procedure which Carathêodory used [3, p. 665] for the case in which $f$ is merely measurable, but in the present context, where $f$ is continuous, this procedure can be made somewhat simpler and can then be described in the following way. Select a sequence $f_{1}, f_{2}, \cdots$ of step-functions which converges to $f$-point-wise convergence almost everywhere is sufficient, although uniform convergence is attainable. Then for each positive integer $n, \operatorname{Re} G\left(x, f_{n}(x)\right)$ as a function of $x$ is measurable by virtue of the measurability of $G(x, w)$ when $w$ is fixed. Because $G(x, w)$ is continuous in $w$ for almost every number $x$ in $(0, a]$, $\operatorname{Re} G(x, f(x))$ is measurable. A similar argument shows that $\operatorname{Im} G(x, f(x))$ is measurable. Therefore $G(x, f(x))$ is measurable.

To obtain the conclusion of Theorem 2 it will be enough to demonstrate that the mapping $U: E \rightarrow C_{0}(0, a]$ defined by $U f=\int_{0+} G(x, f(x)) d x$ is continuous. To this end suppose that each of $f, f_{1}, f_{2}, \cdots$ is a function in $E$ and that $\lim _{n \rightarrow \infty}\left\|f_{n}-f\right\|$ $=0$. Inequality (9) appearing in the proof of Theorem 1 is valid here for the same reasons that it was valid there. From this inequality it follows that if $0<x \leqq a$ and $n$ is a positive integer, then

$$
\begin{aligned}
\left|G\left(x, f_{n}(x)\right)-G(x, f(x))\right| \leqq & \left|G\left(x, f_{n}(x)\right)-G_{0}(x)\right| \\
& +\left|G(x, f(x))-G_{0}(x)\right| \\
\leqq & 2 H(x, t u(x)) .
\end{aligned}
$$

Suppose that $0<b \leqq a$. Since $G(x, w)$ is continuous in $w$ for almost every number $x$ in $(0, a], \lim _{n \rightarrow \infty}\left|G\left(x, f_{n}(x)\right)-G(x, f(x))\right|=0$ for almost every such number 
and in particular for almost every number $x$ in $[b, a]$. Furthermore, since $H(x, t u(x))$ is integrable from $0+$ to $a$ and, consequently, from $b$ to $a$, it follows from the Dominated Convergence Theorem of Lebesgue that

$$
\lim _{n \rightarrow \infty} \int_{b}^{a}\left|G\left(x, f_{n}(x)\right)-G(x, f(x))\right| d x=0 .
$$

Suppose now that $\varepsilon>0$ and that $b$ is small enough that

$$
\left|\int_{0+}^{x} G_{0}\right|+\int_{0+}^{x} H(y, t u(y)) d y<\frac{\varepsilon}{4}
$$

when $0<x \leqq b$. The argument in the proof of Theorem 1 which established that $T(M)$ was a subset of $M$ applies here and shows that $U(E) \subseteq E$, so that each of $U f, U f_{1}, U f_{2}, \cdots$ belongs to $E$. From the defining inequality, (6), for $E$ and the the fact that $f(0+)=0$ it follows that if $0<x \leqq b$, then

$$
|(U f)(x)| \leqq\left|\int_{0+}^{x} G_{0}\right|+\int_{0+}^{x} H(y, t u(y)) d y<\frac{\varepsilon}{4} .
$$

A similar argument shows that if $n$ is a positive integer, then $\left|\left(U f_{n}\right)(x)\right|<\varepsilon / 4$ when $0<x \leqq b$. Suppose now that $N$ is a positive number and is large enough that

$$
\int_{b}^{a}\left|G\left(x, f_{n}(x)\right)-G(x, f(x))\right| d x<\frac{\varepsilon}{2}
$$

whenever $n \geqq N$. If $n$ is such an integer, then, when $0<x \leqq b$,

$$
\begin{aligned}
\left|\left(U f_{n}-U f\right)(x)\right| & \leqq\left|\left(U f_{n}\right)(x)\right|+|(U f)(x)| \\
& <\frac{\varepsilon}{4}+\frac{\varepsilon}{4}=\frac{\varepsilon}{2}<\varepsilon
\end{aligned}
$$

and, when $b<x \leqq a$,

$$
\begin{aligned}
\left|\left(U f_{n}-U f\right)(x)\right| & \leqq\left|\left(U f_{n}-U f\right)(b)\right|+\int_{b}^{x}\left|G\left(y, f_{n}(y)\right)-G(y, f(y))\right| d y \\
& <\frac{\varepsilon}{2}+\frac{\varepsilon}{2}=\varepsilon
\end{aligned}
$$

and therefore $\left\|U f_{n}-U f\right\|<\varepsilon$.

$U$ being thus continuous, there must according to Theorem 1 be a function $f$ in $E$ such that $f=U f=\int_{0+} G(x, f(x)) d x$. The inequalities asserted relating $f$ and the number $t$ follow from the fact that $f \in E$ and from Theorem 1 in that order. -End of proof.

It is part of the theory of Lebesgue integration that if $f=\int_{0+} G(x, f(x)) d x$, then $f$ is differentiable almost everywhere, the improper sense in which the 
integral is taken notwithstanding, and $f^{\prime}(x)=G(x, f(x))$ for almost every number $x$ in the domain of $f$; furthermore, if $G$ is continuous, then $f$ is differentiable everywhere and $f^{\prime}(x)=G(x, f(x))$.

4. Illustrations of Theorem 2. For each of the examples described earlier (ii) of the hypothesis of Theorem 2 is satisfied, and in each case the question whether $G$ is restricted in growth somewhere near 0 , so that (i) also is true, presents itself. To answer this affirmatively, it would suffice to show that there is a number $t$ such that $K_{0}(x)+K(x, t) \leqq t$ if $0<x \leqq a$.

Figure 1 is a picture for Examples 1 and 2 of the graphs, $A_{1}$ and $A_{2}$, of $K_{0}(x)+K(x, t)$ with $x$ fixed, and the graph $B$, of the identity function on the interval $[0, \infty)$. Numbers $t$ for which $K_{0}(x)+K(x, t) \leqq t$ are simply abscissas of

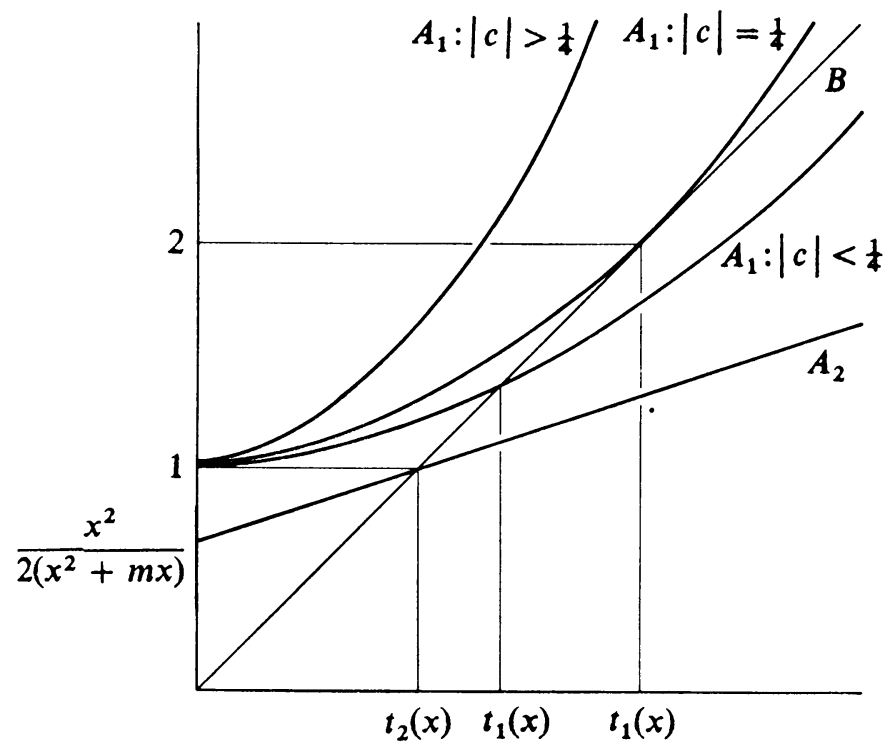

FIGURE 1

points of the appropriate graph, $A_{1}$ or $A_{2}$, lying on or below the graph $B . A_{1}$ has such a point only in case $|c| \leqq 1 / 4$. If $n=1$ and $|c| \leqq 1 / 4$, or $n=2, t_{n}(x)$ is the greatest lower bound of the set of all such numbers $t, x$ remaining fixed, and because of the continuity of $K_{0}$ and $K$ in these particular instances, $K_{0}(x)$ $+K\left(x, t_{n}(x)\right)=t_{n}(x) . t_{1}(x)=2 /\left[1+(1-4|c|)^{1 / 2}\right]$ and $t_{2}(x)=1$, and since neither varies with $x, G$ is restricted in growth somewhere near 0 in Example 1 when $|c| \leqq 1 / 4$ and in Example 2. The lack of dependence of $t_{1}(x)$ upon $x$ in the first example results from the same lack on the part of $K_{0}(x)+K(x, t)$. On the other hand in the second example $K_{0}(x)+K(x, t)$ varies with $x$, and in this case the fact that $t_{2}(x)$ is constant stems from the fact that $u$ is nonnegative and is actually a solution of the integral equation (1). 
Figure 2 is to Examples 3 and 4 as Figure 1 is to Examples 1 and 2. $\left.t_{3}(x)=1 / 2+2[1+9 x / 2)^{1 / 2}\right] / 9 x$, and since in every neighborhood of 0 it is unbounded, there is no solution of $K_{0}(x)+K(x, t) \leqq t$ for Example 3. However, the fact that $A$ and $B$ do alwavs intersect suggests that the integral equation (1)

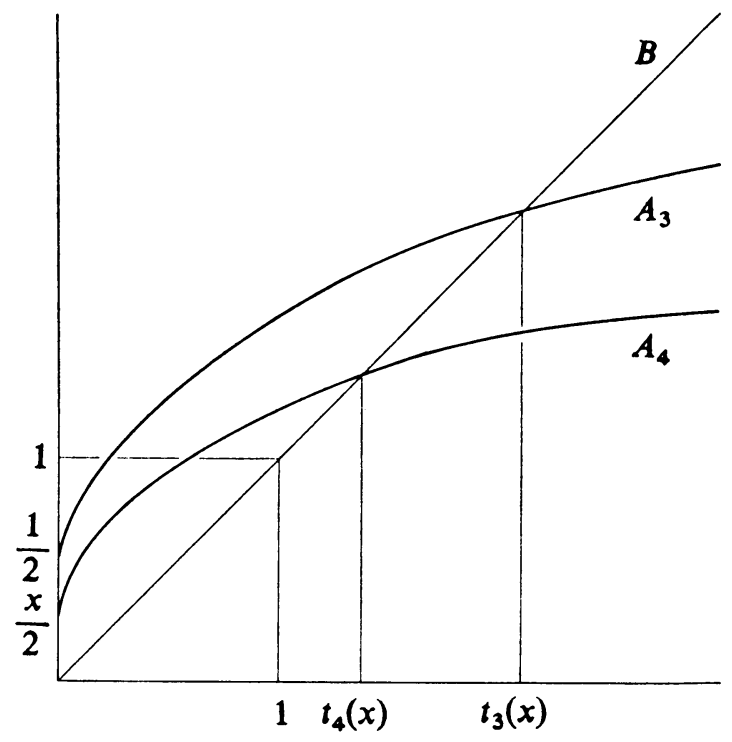

FIGURE 2

might have a solution which is asymptotic at 0 to a multiple of $t_{3}(x) u(x)$. This leads, upon observation that $t_{3}(x) u(x)$ is asymptotic at 0 to $4 x / 9$, to the choice of $x$ for $u(x)$ in Example 4. $t_{4}(x)=\left[1+x+(1+2 x)^{1 / 2}\right] / 2$, and since this is increasing and, for $t$ fixed, $K_{0}(x)+K(x, t)$ is increasing in $x$, it follows that $K_{0}(x)+K\left(x, t_{4}(a)\right) \leqq t_{4}(a)$ when $0<x \leqq a$.

The essence of Theorems 1 and 2 is that the function $G$ is tested by the function $u$ to determine whether there is a multiple of $u, t u$, such that the inequality $\left|\int_{0+}^{x} G(y, t u(y)) d y\right| \leqq t u(x)$ is satisfied near 0 , which, if it is the case, implies with certain additional stipulations that (1) has a solution whose magnitude is not greater than that of $t u$. Because $f(0+)$ is to be 0 , a natural choice for the function $u$ is $\int_{0+} G(x, 0) d x$ or, what is not essentially different, some nonzero multiple thereof. That this choice is not always suitable is demonstrated by Example 3, in which it was the one taken but did not lead to a solution. In this instance as a matter of fact there is no solution which near 0 is dominated by a multiple of $\int_{0+} G(x, 0) d x$, for if $f(x)=x^{2} / 2+\int_{0+}^{x}[|f(y)| / y]^{1 / 2} d y$, then it can be seen inductively that for each nonnegative integer $n, f(x) \geqq s_{n} x^{1+2-n}$, where $s_{0}=1 / 2$ and $s_{n}=s_{n-1}^{1 / 2} /\left(1+2^{-n}\right)$ if $n \geqq 1$, which implies, since $\lim _{n \rightarrow \infty} s_{n}=1$, that $f(x) \geqq x$, whereas $\int_{0+}^{x} G(y, 0) d y=x^{2} / 2$. 
5. Two existence theorems. A direct consequence of Theorem 2 is the next one.

THeOREM 3. Suppose that $0<a<\infty$ and

(i) $G(x, w)$ is measurable as a function of $x$ when $w \in D$, and for almost every number $x$ in $(0, a]$ is continuous as a function of $w$,

(ii) there is a function $A:(0, a] \rightarrow[0, \infty]$ which is integrable from $0+$ to $a$ and is such that $|G(x, w)| \leqq A(x)$ when $x \in(0, a]$ and $w \in D$, and

(iii) $A$ denotes such a function and $a$ is small enough for $\int_{0+}^{a} A$ to belong to $I$.

Then there is a function $f$ in $C_{0}(0, a]$ such that $f=\int_{0+} G(x, f(x)) d x$, $|f(q)-f(p)| \leqq \int_{p}^{q} A$ if $0<p \leqq q \leqq a$, and $|f(x)| \leqq \int_{0+}^{x} A$ if $0<x \leqq a$.

Proof. Let $G_{0}(x)=0, u(x)=\int_{0+}^{x} A, K_{0}(x)=0$ and $H(x, r)=A(x)$, when $x \in(0, a]$ and $r \in I$. Hypothesis A through part (iii) is confirmed by these functions, inequalities (2) and (3) being verified as follows:

and

$$
\left|\int_{0+}^{x} G_{0}\right|=0=K_{0}(x) u(x)
$$

$$
\left|G(x, w)-G_{0}(x)\right|=|G(x, w)| \leqq A(x)=H(x,|w|) .
$$

Inasmuch as $H(x, r)=A(x)$ if $r \in I$, the only restriction upon a nonnegative number $t$ required to ensure that $H(x, t u(x))$ is integrable from $0+$ to $a$ is that $t u(x) \in I$ when $x \in(0, a]$. This restriction is met if $t \leqq 1$, for then $t u(x) \leqq u(x)$ $=\int_{0+}^{x} A \leqq \int_{0+}^{a} A$, which by hypothesis belongs to $I$. The set $J$ therefore includes the closed interval $[0,1]$.

Now let $K(x, t)=1$ if $x \in(0, a]$ and $t \in J$. Hypothesis $A$ is then confirmed by $G_{0}, u, K_{0}, H$ and $K$, for if $x \in(0, a]$ and $t \in J$, then

$$
\int_{0+}^{x} H(y, t u(y)) d y=\int_{0+}^{x} A(y) d y=u(x)=K(x, t) u(x),
$$

so that (4) is true. Furthermore, if $t \in J$ and is 1 or greater, then $K_{0}(x)+K(x, t)$ $=1 \leqq t$. $G$ therefore is restricted in growth somewhere near 0 .

Finally, since the same properties of $G$ having to do with measurability and continuity have been assumed here as were assumed in Theorem 2 , the hypothesis of that theorem is fully satisfied. Its conclusion readily implies the conclusion of Theorem 3, the number $t$ taken in this instance to be 1.-End of proof.

This theorem generalizes an existence theorem of Carathéodory [3], which is, except for minor details, equivalent to the special case of this theorem in which $A$ is integrable on the interval $(0, a]$, rather than merely integrable from $0+$ to $a$.

Also, from Theorem 3 it follows that if $G$ is continuous, $|G|$ is bounded above, by $M$ say, $r$ is the radius of $D$ and is finite, and $L$ is the open interval $(0, r / M)$ if $D$ is open, but is the half-open interval $(0, r / M]$ if $D$ is closed, then there is a differentiable function $f$ with domain $L$ such that $f(0+)=0$ and $f^{\prime}(x)=G(x, f(x))$ if $x \in L$. A proof is obtained by taking $A(x)$ to be $M$. This result generalizes the 
Peano Existence Theorem [7], which in the present context would read the same way as this one except that the additional assumption would be required that $G$ may be extended continuously from the nonclosed set $(0, \infty) \times D$ to the closed set $[0, \infty) \times D$.

The next theorem, in which $G$ is supposed to satisfy a rather weak condition of Lipschitz type, but only at the points $(x, w)$ of the domain of $G$ for which $w=0$, is also a corollary of Theorem 2. If $0<a<\infty$ and $f \in C_{0}(0, a]$, then $\|f\|$ will stand for a function-it will be defined by $\|f\|(x)=\max _{0<y \leqq x}|f(y)|$ if $0<x \leqq a$, and will therefore be a nondecreasing element of $C_{0}(0, a]$ (note that this definition gives a meaning to the symbol $\|f\|$ which differs from its earlier meaning). Also, the conventions that $0^{\circ}=0$ and $1 / 0=0$ will be in force. The latter of these, which is somewhat out of the ordinary, is useful in connection with (iii) (b) of the theorem.

THEOREM 4. Suppose that $0<b<\infty$ and

(i) $G(x, w)$ is measurable as a function of $x$ when $w \in D$, and for almost every number $x$ in $(0, b]$ is continuous as a function of $w$,

(ii) $G(x, 0)$ as a function of $x$ is integrable from $0+$ to $b$,

(iii) there exist a measurable function $A:(0, b] \rightarrow[0, \infty]$ and $a$ bounded measurable function $n:(0, b] \rightarrow[0, \infty]$ such that

(a) $|G(x, w)-G(x, 0)| \leqq A(x)|w|^{n(x)}$ when $x \in(0, b]$ and $w \in D$, and

(b) $A\left\|\int_{0+} G(x, 0) d x\right\|^{n-1}$ is integrable from $0+$ to $b$.

Then there is a function $f$ such that $f=\int_{0+} G(x, f(x)) d x$ and for which there exist a number $a$ in $(0, b]$ and a finite nonnegative number $t$ such that $f \in C_{0}(0, a]$ and $|f(x)| \leqq t\left\|\int_{0+} G(y, 0) d y\right\|(x)$ if $0<x \leqq a$.

Proof. Suppose that $A$ and $n$ are as described, that $N \geqq n(x)$ for every number $x$ in $(0, b]$, and that $a \in(0, b]$ and is small enough for $\left\|\int_{0+} G(x, 0) d x\right\|(a)$ to belong to $I$. Let $m=\int_{0+}^{a} A\left\|\int_{0+}^{a} G(x, 0) d x\right\|^{n-1}$ and suppose further that in case $N=1, a$ is small enough for the inequality $m<1$ to hold, and in case $N>1, a$ is small enough for $m \leqq(N-1)^{N-1} / N^{N}$ to hold. Each of these inequalities is, in the case for which it is designed, necessary and sufficient for there to exist a finite nonnegative number $t$ such that $1+m t^{N} \leqq t$. In case $N<1$ no such inequality is required. If $N=1$, then $t$ must be $1 /(1-m)$ or greater, while if $N>1$, then $t$ may be $N /(N-1)$, but can be smaller unless the inequality is an equality. (These facts are represented in Figure 3.)

Let $G_{0}(x)=G(x, 0), \quad u(x)=\left\|\int_{0+} G(y, 0) d y\right\|(x), \quad K_{0}(x)=1, \quad$ and $H(x, r)$ $=A(x) r^{n(x)}$ if $x \in(0, a]$ and $r \in I$. Then Hypothesis A through part (iii) is confirmed by these functions. The validity of (2) and (3) is established by

$$
\left|\int_{0+}^{x} G_{0}\right| \leqq\left\|\int_{0+} G_{0}\right\|(x)=\left\|\int_{0+} G(y, 0) d y\right\|(x)=u(x)=K_{0}(x) u(x)
$$

and 


$$
\begin{aligned}
\left|G(x, w)-G_{0}(x)\right| & =|G(x, w)-G(x, 0)| \\
& \leqq A(x)|w|^{n(x)} \\
& =H(x,|w|),
\end{aligned}
$$

while (iii) (c) is seen to be true by virtue of the fact that $H(x, 0 \cdot u(x))=H(x, 0)$ $=A(x) \cdot 0^{n(x)}=A(x) \cdot 0=0$ and is, therefore, integrable from $0+$ to $a$.

The next step is to show that $u^{n}$ is measurable on $(0, a]$. Let $c=a$ if $u(a)=0$, but let $c=\inf \{x \mid u(x)>0\}$ if $u(a)>0$. If $c>0$, then, since $u$ is nonnegative and nondecreasing, $u(x)=0$ when $0<x \leqq c$, so that $[u(x)]^{n(x)}=0^{n(x)}=0$ and is measurable on the interval $(0, c]$. If $c<a$, let $g(x, r)=r^{n(x)}$ when $c<x \leqq a$ and $0<r<\infty$. When $r$ is fixed, $g(x, r)$ is measurable in $x$, being a continuous function of the measurable function $n$. When $x$ is fixed, $g(x, r)$ is continuous in $r$, since $r$ cannot be 0 . Because $u$ is continuous, the procedure used by Carathéodory in [3]

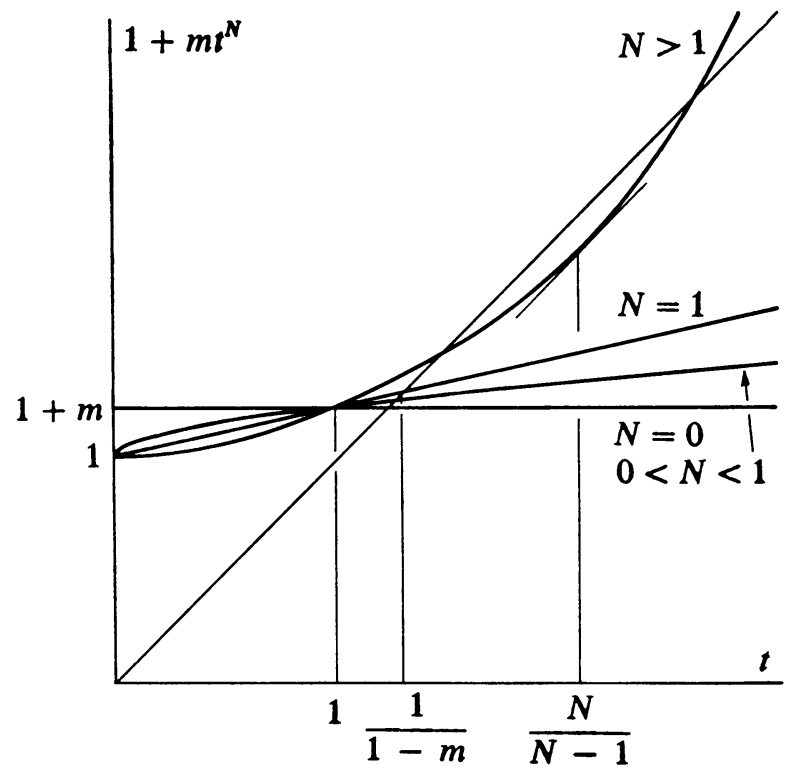

FIGURE 3

and adopted into the proof of Theorem 2 to establish that $G(x, f(x))$ was measurable for every function $f$ in $E$ can be applied to show that $g(x, u(x))$ is measurable, in other words that $[u(x)]^{n(x)}$ is measurable on the interval $(c, a]$. Consequently, $u^{n}$ is measurable on the whole interval $(0, a]$. Noitce also that a similar argument shows that if $t \geqq 0$, then $t^{n}$ is measurable.

If $0 \leqq t \leqq 1$ and $x \in(0, a]$, then $t u(x) \leqq u(x) \leqq u(a)=\left\|\int_{0+} G(y, 0) d y\right\|(a)$, so that $t u(x) \in I$ because $\left\|\int_{0+} G(y, 0) d y\right\|(a) \in I$, and also 


$$
\begin{aligned}
H(x, t u(x)) & =A(x)[t u(x)]^{n(x)} \\
& =A(x) t^{n(x)}[u(x)]^{n(x)} \\
& \leqq A(x)[u(x)]^{n(x)} \\
& =A(x)[u(x)]^{n(x)-1} u(x) \\
& \leqq A(x)\left[\left\|\int_{0+} G(y, 0) d y\right\|(x)\right]^{n(x)-1} u(a) .
\end{aligned}
$$

This shows that if $0 \leqq t \leqq 1$, then $H(x, t u(x))$ as a function of $x$ is measurable (because each of $A, t^{n}$ and $u^{n}$ is measurable on $\left.(0, a]\right)$, is dominated by a function which is integrable from $0+$ to $a$, and, being nonnegative, is itself integrable from $0+$ to $a$. Thus $J$ includes the closed interval $[0,1]$.

Next define $K(x, t)$ for $x$ in $(0, a]$ and $t$ in $J$ by

$$
K(x, t)= \begin{cases}m & \text { if } 0 \leqq t<1, \\ m t^{N} & \text { if } 1 \leqq t .\end{cases}
$$

Then when $x \in(0, a]$ and $t \in J$,

$$
\begin{aligned}
\int_{0+}^{x} H(y, t u(y)) d y & =\int_{0+}^{x} A(t u)^{n} \\
& \leqq\left(\int_{0+}^{a} A t^{n} u^{n-1}\right) u(x) \\
& \leqq \begin{cases}\left(\int_{0+}^{a} A u^{n-1}\right) u(x) & \text { if } 0 \leqq t<1, \\
\left(\int_{0+}^{a} A u^{n-1}\right) t^{N} u(x) & \text { if } 1 \leqq t,\end{cases} \\
& =K(x, t) u(x),
\end{aligned}
$$

since $\int_{0+}^{a} A u^{n-1}=m$. Therefore Hypothesis $\mathrm{A}$ is confirmed by the functions $G_{0}, u, K_{0}, H$ and $K$. Since $K_{0}(x)+K(x, t)=1+m t^{N}$ when $t \geqq 1$, in view of the choice of $a$ there must be a number $t$ such that $K_{0}(x)+K(x, t) \leqq t$, so that $G$ is restricted in growth somewhere near 0 . The hypothesis of Theorem 2 is thereby satisfied, and its conclusion implies the desired conclusion. - End of proof.

6. Uniqueness, but not of a solution. When a global Lipschitz condition is utilized to ensure existence of a solution of the initial-value problem, a common by-product is the conclusion that there can be no other solution. Well-known 
examples show that uniqueness of the solution is not deducible from the local Lipschitz condition postulated in Theorem 4. However, it can be inferred from the hypothesis of Theorem 4 , that, loosely speaking, every solution $f$ satisfying $|f(x)| \leqq t\left\|\int_{0_{+}} G(y, 0) d y\right\|(x)$ must be asymptotic at 0 to the function $\int_{0_{+}} G(x, 0) d x$, in other words that $\lim _{x \rightarrow 0}\left[f(x) / \int_{0+}^{x} G(y, 0) d y\right]=1$ for every such solution $f$ and is therefore "unique". This is expressed accurately in the next theorem, for which will be maintained the conventions that $0^{\circ}=0$ and $1 / 0=0$.

THEOREM 5. Suppose that the hypothesis of Theorem 4 is true, let $u=\left\|\int_{0+} G(x, 0) d x\right\|$, let $c=b$ if $u(b)=0$, and let $c=\inf \{x \mid u(x)>0\}$ if $u(b)>0$. If $c>0$, then $\int_{0+}^{x} G(y, 0) d y=0$ when $0<x \leqq c$, and if $f$ verifies the conclusion of Theorem 4, then $f(x)=0$ when $0<x \leqq c$. If $c=0$, and to the hypothesis of Theorem 4 is added

$$
\lim _{x \rightarrow 0 ; x \in M} \frac{\left(\int_{0+}^{x} A u^{n-1}\right) u(x)}{\left|\int_{0+}^{x} G(y, 0) d y\right|}=0,
$$

where $M=\left\{x \mid \int_{0+}^{x} G(y, 0) d y \neq 0\right\}$, then if $f$ verifies the conclusion of Theorem 4,

$$
\lim _{x \rightarrow 0 ; x \in M} \frac{f(x)}{\int_{0+}^{x} G(y, 0) d y}=1 .
$$

Proof. The first statement of the conclusion is obvious, so consider that $c=0$ and that (iii)(c) is true. Suppose that $f$ verifies the conclusion of Theorem 4 and that $a$ and $t$ are related to $f$ in the way described in that conclusion. Suppose further that $N \geqq n(x)$ for every number $x$ in $(0, a]$. If $0<x \leqq a$, then

$$
\begin{aligned}
\left|f(x)-\int_{0+}^{x} G(y, 0) d y\right| & \leqq \int_{0+}^{x}|G(y, f(y))-G(y, 0)| d y \\
& \leqq \int_{0+}^{x} A(y)|f(y)|^{n(y)} d y \\
& \leqq \int_{0+}^{x} A t^{n} u^{n} \\
& \leqq \begin{cases}\left(\int_{0+}^{x} A u^{n-1}\right) u(x) & \text { if } 0 \leqq t<1, \\
\left(\int_{0+}^{x} A u^{n-1}\right) t^{N} u(x) & \text { if } 1 \leqq t,\end{cases}
\end{aligned}
$$

convergence of the first three integrals on the right being a consequence of the convergence of the last one. Therefore if $0<x \leqq a$ and $x \in M$, then 


$$
\left|\frac{f(x)}{\int_{0+}^{x} G(y, 0) d y}-1\right| \leqq \begin{cases}\frac{\left(\int_{0+}^{x} A u^{n-1}\right) u(x)}{\left|\int_{0+}^{x} G(y, 0) d y\right|} & \text { if } 0 \leqq t<1, \\ \frac{\left(\int_{0+}^{x} A u^{n-1}\right) u(x)}{\left|\int_{0+}^{x} G(y, 0) d y\right|} t^{N} & \text { if } 1 \leqq t .\end{cases}
$$

The conclusion of the theorem is implied by this inequality and the condition (iii)(c).-End of proof.

7. Restrictedness in growth of $G$ somewhere near 0 as a consequence of solvability of $f=\int_{0+} G(x, f(x)) d x$. In order that the integral equation (1) have a solution it is not in general necessary that $G$ be restricted in growth somewhere near 0 . This results primarily from the fact that the function $H$ of Hypothesis A dominates $G$ throughout the subset $(0, a] \times D$ of its domain, whereas existence of a solution $f$ of (1) restricts $G$ only along the path traced in this subset by the point $(x, f(x))$ as $x$ varies, while allowing $G$ to behave elsewhere in this subset in a completely willful manner. However, some additional restrictions can be put upon $G$ which will make it orderly enough to allow the conclusion that if (1) has a real-valued solution, then $G$ is restricted in growth somewhere near 0 . This is shown by the next theorem.

THEOREM 6. Suppose that $0<b<\infty$ and

(i) $G(x, w) \geqq 0$ when $x \in(0, b], w \in D$ and $w$ is real,

(ii) $G\left(x, r_{1}\right) \leqq G\left(x, r_{2}\right)$ when $x \in(0, b], r_{1} \in I, r_{2} \in I$ and $r_{1} \leqq r_{2}$, and

(iii) $|G(x, w)| \leqq G(x,|w|)$ when $x \in(0, b]$ and $w \in D$.

If there exist a positive number $a$ and a real-valued function $f$ on $(0, a]$ such that $f=\int_{0+} G(x, f(x)) d x$, then $G$ is restricted in growth somewhere near 0.

Proof. Suppose that $a \leqq b$; this incurs no loss of generality. Since $f(x)$ is always real, $G(x, f(x)) \geqq 0$, and consequently $f$, being the same as $\int_{0+} G(x, f(x)) d x$, must be nonnegative and nondecreasing.

Let $G_{0}(x)=0, u(x)=f(x), K_{0}(x)=0$ and $H(x, r)=G(x, r)$ when $x \in(0, a]$ and $r \in I$. The conditions of Hypothesis A through (iii)(a) are readily seen to be met by these functions. If $x \in(0, a]$ and $w \in D$, then

$$
\left|G(x, w)-G_{0}(x)\right|=|G(x, w)| \leqq G(x,|w|)=H(x,|w|),
$$

and therefore (iii)(b) is satisfied. If $t=1$ and $0<x \leqq a$, then $t u(x)=f(x)$, which must belong to $I$, and 


$$
H(x, t u(x))=G(x, t u(x))=G(x, f(x)),
$$

which is integrable from $0+$ to $a$. This shows that (iii)(c) is satisfied and that $1 \in J$.

Now define a function $K$ in the following way: if $x \in(0, a]$ and $t \in J$, let

$$
K(x, t)= \begin{cases}\frac{\int_{0+}^{x} H(y, t u(y)) d y}{u(x)} & \text { if } u(x) \neq 0, \\ 1 & \text { if } u(x)=0 .\end{cases}
$$

Suppose that $x \in(0, a]$ and $t \in J$. Clearly, $\int_{0+}^{x} H(y, t u(y)) d y \leqq K(x, t) u(x)$ if $u(x) \neq 0$. If $u(x)=0$, then because $f$, and therefore $u$, is nonnegative and nondecreasing, $f(y)=u(y)=0$ for $0<y \leqq x$, and so

$$
\begin{aligned}
\int_{0+}^{x} H(y, t u(y)) d y & =\int_{0+}^{x} G(y, t u(y)) d y=\int_{0+}^{x} G(y, 0) d y \\
& =\int_{0+}^{x} G(y, f(y)) d y=f(x)=0=K(x, t) u(x) .
\end{aligned}
$$

Therefore Hypothesis $\mathrm{A}$ is confirmed by the functions $G_{0}, u, K_{0}, H$ and $K$.

As was noticed earlier, $1 \in J$. If $x \in(0, a]$ and $u(x) \neq 0$, then

$$
\int_{0+}^{x} H(y, 1 u(y)) d y=\int_{0+}^{x} G(y, f(y)) d y=f(x)=u(x),
$$

so that $K(x, 1)=1$; if on the other hand $u(x)=0$, then $K(x, 1)=1$ by definition. Therefore there is a number $t$ which satisfies inequality (5), since $K_{0}(x)+K(x, 1)$ $=0+1 \leqq 1$. - End of proof.

8. Unsolvability of $f=\int_{0+} G(x, f(x)) d x$ as a consequence of forced growth of $G$ somewhere near 0 . The preceding theorem says, approximately, that Hypothesis $A$ and existence of a solution of (5) are implied by solvability of (1), and thus is an approximate converse of the principal idea expressed in Theorem 1 and again in Theorem 2. Another way to turn that idea around would be to obtain a new condition upon $G$ from Hypothesis $A$ by reversing its inequalities and then show that this new hypothesis and nonexistence of a solution of (5) imply that (1) is not solvable. Most of the remainder of the paper will be devoted to doing something of this nature. Hypothesis A will be displaced by the following statement.

HyPOTHESIS B. There exist a finite positive number $a$, a function $G_{0}:(0, a] \rightarrow P_{\infty}$, a nonnegative function $u$ on $(0, a]$, a nonnegative number $K_{0}$ and a nonnegative function $H$ on $(0, a] \times I$, such that

(i) if $b \in(0, a]$, then there is a number $x$ such that $0<x \leqq b$ and $u(x) \neq 0$,

(ii) if $x \in(0, a]$ and $G_{0}$ is integrable from $0+$ to $x$, then

$$
\left|\int_{0+}^{x} G_{0}\right| \geqq K_{0} u(x),
$$


(iii) (a) for each number $x$ in $(0, a], H(x, r)$ is nondecreasing in $r$, (b) if $x \in(0, a]$ and $w \in D$, then

$$
\left|G(x, w)-G_{0}(x)\right| \geqq H(x,|w|),
$$

and

(c) if $t \geqq 0,0<x \leqq a$, and $t u(y) \in I$ when $0<y \leqq x$, then $H(y, t u(y))$ as a function of $y$ on the interval $(0, x]$ is measurable, and

(iv) with $J$ signifying the set of which $t$ is an element only if $t \geqq 0$ and for some number $x$ in $(0, a], t u(y) \in I$ when $0<y \leqq x$, there exists a nonnegative function $K$ on $J$ such that if $t \in J$ and $x$ is such a number, then either $H(y, t u(y))$ is not integrable from $0+$ to $x$, or else it is and

$$
\int_{0+}^{x} H(y, t u(y)) d y \geqq K(t) u(x) .
$$

Hypothesis A and Hypothesis B are not incompatible with each other. For instance, if $a$ is taken to be any finite positive number, and functions $G_{0}, u, H$ and $K$ and a number $K_{0}$ are so defined that $G_{0}(x)=1, u(x)=x, K_{0}=1, H(x, r)$ $=|c|(r / x)^{2}$ and $K(t)=|c| t^{2}$, then they confirm Hypothesis B for Example 1. In a similar manner Hypothesis B can be shown to hold for Example 2 and for Examples 3 and 4.

Just as in the case of Hypothesis A, if $G_{0}, u, K_{0}, H$ and $K$ meet the conditions of Hypothesis B, then $K_{0}, H$ and $K$, if they do not have the property of being the "best possible", may be replaced by $K_{0}^{*}, H^{*}$ and $K^{*}$, which do have this property, being defined by

and

$$
\begin{aligned}
K_{0}^{*} & =\inf _{x \in(0, a]} \frac{\left|\int_{0+}^{x} G_{0}\right|}{u(x)}, \\
H^{*}(x, r) & =\inf _{|w| \geq r}\left|G(x, w)-G_{0}(x)\right|
\end{aligned}
$$

$$
K^{*}(t)=\inf _{x \in(0, a]} \frac{\int_{0+}^{x} H^{*}(y, t u(y)) d y}{u(x)},
$$

where each of the ratios stands for $\infty$ if either $u(x)=0$ or the expression in its numerator fails to have meaning. "Best possible" in this context is meant to imply that $K_{0}^{*}, H^{*}(x, r)$ and $K^{*}(t)$ are as large as they can be-so that the inequality $K_{0}^{*}+K^{*}(t) \leqq t$ is least likely to be satisfiable-without destroying one of inequalities (11), (12) and (13) or failing to satisfy the other applicable conditions of Hypothesis B. Here, similarly as for Hypothesis A, this "best possible" choice is not available if $H^{*}(y, t u(y))$ turns out not to be measurable on any interval $(0, x]$ with $0<x \leqq a$. 
Now a notion complementary to that of $G$ 's being restricted in growth somewhere near 0 can be defined.

Definition 2. The function $G:(0, \infty) \times D \rightarrow P_{\infty}$ is said to be forced to grow somewhere near 0 if and only if Hypothesis $B$ is true and for some set $\left\{G_{0}, u, K_{0}, H, K\right\}$ which confirms Hypothesis B there is no finite number $t$ such that

$$
K_{0}+K(t) \leqq t
$$

THEOREM 7. Suppose that $G$ is forced to grow somewhere near 0 , and $G_{0}, u, K_{0}, H$, and $K$ constitute a set of functions and a number $K_{0}$ which together confirm this fact. Then there do not exist a number $b$ in the interval $(0, a]$ and $a$ function $f:(0, b] \rightarrow P$ such that

$$
|f(x)| \geqq\left|\int_{0+}^{x} G_{0}\right|+\int_{0+}^{x}\left|G(y, f(y))-G_{0}(y)\right| d y
$$

when $0<x \leqq b$; in particular if $G_{0}$ is integrable from $0+$ to $a$ and $\int_{0+}^{x} G_{0}$ and $G(x, w)-G_{0}(x)$ are either both nonnegative whenever $x \in(0, a]$ and $w \in D$ or both nonpositive when $x \in(0, a]$ and $w \in D$, then there do not exist a number $b$ in the interval $(0, a]$ and a function $f:(0, b) \rightarrow P$ such that $f=\int_{0^{+}} G(x, f(x)) d x$.

Proof. Suppose that the conclusion is false. Let $b$ stand for a number in the interval $(0, a]$ and $f$ for a function from the interval $(0, b]$ into $P$, and suppose that (15) is true when $0<x \leqq b$. There is at least one nonnegative number $t$, for example 0 , for which $|f(x)| \geqq t u(x)$ when $0<x \leqq b$. Let $M=\{t \mid t \geqq 0$ and $|f(x)| \geqq t u(x)$ when $0<x \leqq b\}$ and let $T=\sup M$. Clearly, $T<\infty$, since $u(x)>0$ for at least one number $x$ in the interval $(0, b]$. Also, $T \in M$, for if $x \in(0, b]$, then $|f(x)| \geqq \sup _{t \in M} t u(x)=T u(x)$.

Suppose that $0<x \leqq b$. A part of the assumption that (15) holds is that $G_{0}$ is integrable from $0+$ to $x$, which implies that (11) is true. Another part of this assumption is that if $0<y \leqq x$, then $f(y) \in D$, which implies that $|f(y)| \in I$, so that $T u(y)$, being not greater than $|f(y)|$, belongs to $I$. Consequently, $T \in J$.

If $0<y \leqq x$, then from (12) and the nondecreasing character of $H(y, r)$ it follows that

$$
\left|G(y, f(y))-G_{0}(y)\right| \geqq H(y,|f(y)|) \geqq H(y, T u(y)) .
$$

Since $H(y, T u(y))$ as a function of $y$ on the interval $(0, x]$ is nonnegative and measurable, and according to the last inequalities is dominated by a function which, again as part of the assumption that (15) holds, is integrable from $0+$ to $x$, it must itself be integrable from $0+$ to $x$, and therefore (13) is true when $t=T$. Now a combined use of (11), (16) and (13) leads from the fact that (15) holds to the conclusion that 


$$
\begin{aligned}
|f(x)| & \geqq K_{0} u(x)+\int_{0+}^{x} H(y, T u(y)) d y \\
& \geqq K_{0} u(x)+K(T) u(x) \\
& =\left[K_{0}+K(T)\right] u(x) .
\end{aligned}
$$

But this implies that $K_{0}+K(T) \in M$, so that $K_{0}+K(T) \leqq T$, which is a contradiction.

The fact that the integral inequality (15) has no local solution near 0 is thus a consequence of the hypothesis of the theorem. The remainder of the conclusion of the theorem follows readily from this fact.-End of proof.

9. Remarks. If the initial condition is that $f(0+)=c$, a complex number, then the preceding theorems may be applied to the function $G^{*}$ defined by $G^{*}(x, w)$ $=G(x, w+c)$, inasmuch as the integral equation $f^{*}=\int_{0+} G^{*}\left(x, f^{*}(x)\right) d x$ is the same as the equation $f=c+\int_{0+} G(x, f(x)) d x$ if $f^{*}=f-c$. Similarly, the case in which the domain of $G$ is $(-\infty, 0) \times D$ instead of $(0, \infty) \times D$, and the initial condition is that $f(0-)=c$, can be reduced by an $x$-reflection and a $w$-translation to the one already handled.

With appropriate modifications much of the theory which has been described here can be extended to the case in which $G$, and therefore $f$, takes its values from a finite-dimensional Banach space, or from an infinite-dimensional Banach space when a suitable notion of integration is at hand.

\section{REFERENCES}

1. G. D. Birkhoff and O. D. Kellogg, Invariant points in function space, Trans. Amer. Math. Soc. 23 (1922), 96-115.

2. L. E. J. Brouwer, Uber Abbildungen von Mannigfaltigkeiten, Math. Ann. 71 (1912), 97-115.

3. C. Carathéodory, Vorlesungen über reelle Funktionen, pp. 665-672, Chelsea, New York, 1948.

4. A. L. Cauchy, Surl'intégration des équations différentielles, Oeuvres Complétes d'Augustin Cauchy, Ser. 2, Vol. 11, pp. 399-465, Gauthier-Villars, Paris, 1913.

5. R. Lipschitz, Sur la possibilité d'intégrer complétement un système donné d'équations différentielles, Bull. Sci. Math. (1) 10 (1876), 149-159.

6. M. Müller, Neuere Untersuchungen über den Fundamentalsatz in der Theorie der gewöhnlichen Differentialgleichungen, Jber. Deutsch. Math.-Verein. 37 (1928), 33-48.

7. G. Peano, Démonstration de l'intégrabilité des équations différentielles ordinaires, Math. Ann. 37 (1890), 182-228.

8. J. Schauder, Der Fixpunktsatz in Funktionalräumen, Studia Math. 2 (1930), 171-180.

UNIVERSITY OF WASHINGTON, SEATtLE, WASHINGTON 